MATHEMATICS OF COMPUTATION

Volume 73, Number 248, Pages 1655-1671

S 0025-5718(03)01619-3

Article electronically published on November 24, 2003

\title{
A LOCKING-FREE REISSNER-MINDLIN QUADRILATERAL ELEMENT
}

\author{
HUO-YUAN DUAN AND GUO-PING LIANG
}

\begin{abstract}
On arbitrary regular quadrilaterals, a new finite element method for the Reissner-Mindlin plate is proposed, where both transverse displacement and rotation are approximated by isoparametric bilinear elements, with local bubbles enriching rotation, and a local reduction operator is applied to the shear energy term. This new method gives optimal error bounds, uniform in the thickness of the plate, for both transverse displacement and rotation with respect to $H^{1}$ and $L^{2}$ norms.
\end{abstract}

\section{INTRODUCTION}

The Reissner-Mindlin plate bending model describes the deflection of a plate with small to moderate thickness subject to a transverse load and allows the use of simple $C^{0}$ approximations for both transverse displacement and rotation.

However, the standard finite element method for the Reissner-Mindlin plate may suffer from the so-called shear locking phenomenon when the thickness of the plate goes to zero. Roughly, when the thickness becomes relatively small, the shear energy term imposes the Kirchhoff constraint, resulting in almost zero displacements; cf. [19], 14], 8]. Therefore, how to design locking-free elements has been and still remains an active research subject, and a lot of elements have been proposed and good results have been reported, where the general approach to avoid the shearlocking phenomenon is to introduce a reduction operator into the shear energy term; cf. [3], [5], [1], [6], 7], [10]-17], [22]-30], 35], [38]-40], etc.

The MITC4 (Bathe-Dvorkin) element is such an element, and shows superior performance; cf. 11, [29, 20]. However, only for a very restricted class of meshes, this element and its stabilized variants were shown to be suboptimally or optimally convergent, uniformly with respect to the plate thickness; see [2], 32], 3], [4]. These restrictions on meshes are similar to those on $\mathcal{Q}_{1}-\mathcal{Q}_{0}$ element for the Stokes equation (cf. 31, 8, 34) and those on the nonconforming quadrilateral Wilson element (cf. 21]). In general, all these elements do not yield good approximations on arbitrary regular quadrilaterals.

The purpose of this paper is to propose a new quadrilateral finite element method for the Reissner-Mindlin plate, where both transverse displacement and rotation are approximated by isoparametric bilinear elements, with local bubbles enriching the rotation, and the shear stress is obtained by a local reduction operator onto

Received by the editor July 19, 2002 and, in revised form, March 17, 2003.

2000 Mathematics Subject Classification. Primary 65N30.

Key words and phrases. Reissner-Mindlin plates, finite element method.

(C)2003 American Mathematical Society 
a discontinuous quadrilateral variant of the rotated lowest-order Raviart-Thomas rectangular element (cf. [8]).

This new method can accommodate arbitrary regular quadrilaterals in the usual sense (cf. [18]), benefitting from the quadrilateral variant constructed by [40], [33] of the rectangular Raviart-Thomas element of lowest order.

This new method may be viewed as a generalization of the MITC4 element, since the reduction operator is a combination of the local interpolation operator $\Pi_{h}$ for $H_{0}(\operatorname{curl} ; \Omega)$ and the local $L^{2}$ projection operator $\Pi_{h}^{0}$, where $\Pi_{h}$ is applied to the bilinear part of the rotation and $\Pi_{h}^{0}$ is applied to the bubble part of the rotation. As a whole, the shear stress is discontinuous, in contrast with the tangential continuity in the MITC4 element.

Following a general argument by [3] and a simplified version by [5], we show that this new method gives optimal convergence in $H^{1}$-norm for transverse displacement and rotation, uniform in the thickness of the plate. Therefore, this new method is locking-free.

Moreover, following the classical Aubin-Nitsche duality argument, we obtain the uniform optimal $L^{2}$ error bounds for both transverse displacement and rotation, with the help of the Helmholtz-decomposition for the space $\left(L^{2}(\Omega)\right)^{2}$.

As far as we know, the general proof to show the $L^{2}$ error bounds for the MITC4 element has been missing for many years. The main difficulty lies in how to estimate the inconsistency term. For the MITC3 triangular element 3], 29], an approach was given in [37]. Regarding higher-order MITC elements, the proof is basically trivial (see [7, [15], [35]). However, the argument in 37], [7], [15], 35] cannot be applied to the lowest-order rectangular or quadrilateral Reissner-Mindlin elements such as the MITC4 element and its stabilized variants.

The argument we have developed for the new method of this paper is also valid for the MITC4 element and its stabilized variants. Our argument benefits from a local linked interpolation ([25], [26], mathematically analyzed by [38], [39]) which helps us to establish a special Helmholtz-decomposition. Consequently, we can bound the inconsistency term $\left(\mathbf{q}, \tilde{\beta}-\Pi_{h} \tilde{\beta}\right)$ by $\mathcal{O}\left(h^{2}\right)$.

The rest of this paper is organized as follows. In Section 2 we recall the ReissnerMindlin plate model and the MITC4 element and the quadrilateral variant of the rectangular Raviart-Thomas element of lowest order. Section 3 is concerned with the new finite element approximation. In Section 4, we obtain error bounds in $H^{1}$-norm. In the last section, we obtain the $L^{2}$ error bounds.

Throughout this paper, the letter $C$ is a generic positive constant which is independent of the plate-thickness $t$ and the parameter $h$ of the triangulation.

\section{Preliminaries}

2.1. The Reissner-Mindlin plate model. Let $\Omega$ be the region occupied by the plate. Let $w$ and $\phi$ denote the transverse displacement of $\Omega$ and the rotation of fibers normal to $\Omega$. Assuming a clamped boundary condition, the Reissner-Mindlin plate model $([8],[23])$ is to find $(w, \phi) \in H_{0}^{1}(\Omega) \times\left(H_{0}^{1}(\Omega)\right)^{2}$ such that

$$
a(\phi, \psi)+(\mathbf{q}, \nabla v-\psi)=(f, v)
$$

and

$$
\mathbf{q}=\lambda t^{-2}(\nabla w-\phi) \in H_{0}(\operatorname{curl} ; \Omega)
$$


for all $(v, \psi) \in H_{0}^{1}(\Omega) \times\left(H_{0}^{1}(\Omega)\right)^{2}$, where $\mathbf{q}$ is known as the shear stress, $f$ is the transverse load, $t$ is the thickness of the plate, $\lambda=E \kappa / 2(1+\nu)$ is the shear modulus with $E$ the Young's modulus, $\nu$ the Poisson ratio, and $\kappa$ the shear correction factor. $a(\phi, \psi)$ is a coercive bilinear form, defined by

$$
a(\phi, \psi)=\frac{E}{12\left(1-\nu^{2}\right)} \int_{\Omega}(1-\nu) \varepsilon(\phi): \varepsilon(\psi)+\nu \operatorname{div} \phi \operatorname{div} \psi
$$

with $\varepsilon(\phi)=\left(\nabla \phi+\nabla \phi^{T}\right) / 2$ the linear strain tensor,

$$
H_{0}(\operatorname{curl} ; \Omega)=\left\{\mathbf{s} \in\left(L^{2}(\Omega)\right)^{2} ; \operatorname{curl} \mathbf{s} \in L^{2}(\Omega), \mathbf{s} \cdot \tau_{\mid \partial \Omega}=0\right\},
$$

where $\tau$ is the unit tangent to the boundary.

Remark 2.1. When $t$ goes to zero, the Reissner-Mindlin plate degenerates into the classical Kirchhoff-Love model, the solution $\left(w_{0}, \phi_{0}\right)$ of which satisfies the wellknown Kirchhoff constraint (cf. [14, [8])

$$
\nabla w_{0}=\phi_{0} .
$$

In the standard displacement-based linear finite element method, for small but nonvanishing $t$, the shear energy term will force the approximations $\left(w_{h}, \phi_{h}\right)$ to nearly satisfy the Kirchhoff constraint, implying that $\left(w_{h}, \phi_{h}\right)$ is nearly zero. This purely numerical phenomenon is known as shear-locking.

2.2. The MITC4 element. Let $\Omega$ be a convex polygon, and let $\mathcal{T}_{h}$ be a triangulation of $\Omega$ into rectangles. Let $S(K):=R T_{[0]}(K)$ be the rotated lowest-order Raviart-Thomas rectangular element (cf. [8]), and let $\mathcal{Q}_{1}(K)$ be the space of bilinear polynomials.

Then, the MITC4 element is defined as follows (cf. [1], [2], [29]):

$$
\begin{gathered}
\mathbf{S}_{h}=\left\{\mathbf{s} \in H_{0}(\operatorname{curl} ; \Omega) ; \mathbf{s}_{\left.\right|_{K}} \in S(K), \forall K \in \mathcal{T}_{h}\right\}, \\
W_{h}=\left\{v \in H_{0}^{1}(\Omega) ; v_{\left.\right|_{K}} \in \mathcal{Q}_{1}(K), \forall K \in \mathcal{T}_{h}\right\}, \\
\mathbf{H}_{h}=\left(W_{h}\right)^{2}
\end{gathered}
$$

Introduce the standard interpolation operator $\Pi_{h}: \chi \in H_{0}(\operatorname{curl} ; \Omega) \cap\left(H^{1}(\Omega)\right)^{2}$ $\rightarrow \Pi_{h} \chi \in \mathbf{S}_{h}$ as follows:

$$
\int_{e}\left(\Pi_{h} \chi-\chi\right) \cdot \tau_{e}=0, \quad \forall \text { edge } e,
$$

where $\tau_{e}$ is the unit tangent to edge $e$. The MITC4 finite element method is to find $\left(w_{h}, \phi_{h}\right) \in W_{h} \times \mathbf{H}_{h}$ such that

$$
a\left(\phi_{h}, \psi\right)+\left(\mathbf{q}_{h}, \nabla v-\psi\right)=(f, v)
$$

and

$$
\mathbf{q}_{h}=\lambda t^{-2}\left(\nabla w_{h}-\Pi_{h} \phi_{h}\right) \in \mathbf{S}_{h}
$$

for all $(v, \psi) \in W_{h} \times \mathbf{H}_{h}$.

Remark 2.2. When the family of meshes $\left\{\mathcal{T}_{h}\right\}$ is obtained by uniform refinement of a starting rectangular mesh in such a way that at each step every element is divided uniformly in sixteen rectangles, [3] obtained a uniform error bound $\mathcal{O}\left(h^{1 / 2}\right)$, where $\phi$ is required to be in $\left(H^{5 / 2}(\Omega)\right)^{2}$. 
For quadrilaterals, in addition to the above similar assumption, assuming that the distance between the midpoints of two diagonals of each quadrilateral $K \in \mathcal{T}_{h}$ is not greater than a constant multiple of $h_{K}^{2}\left(h_{K}\right.$ is the diameter of $\left.K\right)$, [4], [22], [35] proposed stabilized variants of (2.9) and (2.10) and obtained a uniform optimal error bound $\mathcal{O}(h)$. However, in these papers, the $L^{2}$ error bounds are not available for both transverse displacement and rotation.

2.3. A quadrilateral variant of $R T_{[0]}$. Recently, 40], 33] proposed a variant of the lowest-order Raviart-Thomas rectangular element $R T_{[0]}$. This variant can accommodate arbitrary regular quadrilaterals and satisfies the well-known property of commuting diagrams.

Let $\mathcal{T}_{h}$ be the regular triangulation of $\Omega$ into convex quadrilaterals; cf. [18]. $F_{K}: \hat{K}=[-1,1] \times[-1,1] \rightarrow K$ is the standard invertible mapping, with inverse $F_{K}^{-1}$, where $\hat{K}$ is the reference square in the $\xi \eta$-plane.

The rotated version of the quadrilateral flux element constructed by [40], [33] is as follows:

$$
D L(K)=\operatorname{span}\left\{(1,0)^{T},(0,1)^{T},(-y, x)^{T}, \nabla\left(N_{1} \circ F_{K}^{-1}\right)\right\},
$$

where $N_{1}=(1+\xi)(1+\eta) / 4$ is one of local base functions of the isoparametric bilinear space $\mathcal{Q}_{1}(K)$, and the degrees of freedom for $D L(K)$ are the moments on the edges of the tangential components.

Similar to (2.8), with $S(K):=D L(K)$, define $\Pi_{h}$ as

$$
\int_{e}\left(\Pi_{h} \chi-\chi\right) \cdot \tau_{e}=0, \quad \forall \text { edge } e \in \partial K, \forall K \in \mathcal{T}_{h} .
$$

We have the interpolation property

$$
\left\|\chi-\Pi_{h} \chi\right\|_{0} \leq C h|\chi|_{1}
$$

and we have the property of commuting diagrams

$$
\operatorname{curl} \Pi_{h} \chi=P_{h} \operatorname{curl} \chi,
$$

where $P_{h}$ is the standard local $L^{2}$ orthogonal projection operator onto $M_{h}=\{v \in$ $\left.L^{2}(\Omega) ; v_{\left.\right|_{K}} \in \mathcal{P}_{0}(K), \forall K \in \mathcal{T}_{h}\right\}$, with $\mathcal{P}_{0}(K)$ the space of constants.

Remark 2.3. Note that other quadrilateral variants do not satisfy (2.14) generally; cf. [37, [8]. We will use (2.14) to derive the $L^{2}$ error bounds for the new method of this paper.

Define

$$
\Gamma_{h}=\left\{\mathbf{s} \in\left(L^{2}(\Omega)\right)^{2} ; \mathbf{s}_{\left.\right|_{K}} \in S(K), \forall K \in \mathcal{T}_{h}\right\} .
$$

We can introduce a standard local $L^{2}$ projection operator $\Pi_{h}^{0}: \chi \in\left(L^{2}(\Omega)\right)^{2} \rightarrow$ $\Pi_{h}^{0} \chi \in \Gamma_{h}$ as follows:

$$
\left\{\begin{array}{l}
\int_{K}\left(\Pi_{h}^{0} \chi-\chi\right) \mathbf{s}=0, \quad \forall \mathbf{s} \in S(K), \forall K \in \mathcal{T}_{h}, \\
\left\|\chi-\Pi_{h}^{0} \chi\right\|_{0} \leq C h|\chi|_{1}, \quad \text { if } \chi \in\left(H^{1}(\Omega)\right)^{2} .
\end{array}\right.
$$




\section{Finite ELEMENT APPROXIMATION}

In this section, we will propose a new quadrilateral finite element method for problem (2.1) and (2.2).

Let $b_{K}$ be the usual bubble on $K$; i.e., $b_{K} \circ F_{K}=\left(1-\xi^{2}\right)\left(1-\eta^{2}\right) / 16 \in H_{0}^{1}(\hat{K})$, and $S(K):=D L(K)$ defined as in (2.11). We introduce

$$
\begin{gathered}
\mathbf{B}_{h}=\left\{\mathbf{s} ; \mathbf{s}_{\left.\right|_{K}} \in S(K) b_{K} \subset\left(H_{0}^{1}(K)\right)^{2}, \forall K \in \mathcal{T}_{h}\right\}, \\
\mathbf{S}_{h}=\left\{\mathbf{s} \in H_{0}(\operatorname{curl} ; \Omega) ; \mathbf{s}_{\left.\right|_{K}} \in S(K), \forall K \in \mathcal{T}_{h}\right\}, \\
W_{h}=\left\{v \in H_{0}^{1}(\Omega) ; v_{\left.\right|_{K}} \in \mathcal{Q}_{1}(K), \forall K \in \mathcal{T}_{h}\right\}, \\
\mathbf{H}_{h}^{+}=\left(W_{h}\right)^{2} \oplus \mathbf{B}_{h}, \\
\Gamma_{h}=\left\{\mathbf{s} \in\left(L^{2}(\Omega)\right)^{2} ; \mathbf{s}_{\left.\right|_{K}} \in S(K), \forall K \in \mathcal{T}_{h}\right\},
\end{gathered}
$$

where $W_{h}, \mathbf{H}_{h}^{+}, \Gamma_{h}$ will be used for approximating subspaces of transverse displacement and rotation and shear stress, respectively. The degrees of freedom associated with these finite dimensional spaces are depicted in Figures 1 and 2.

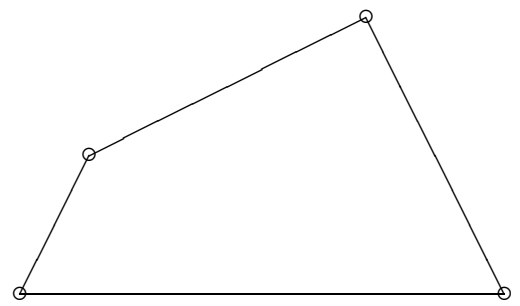

$w-\mathcal{Q}_{1}(K)$

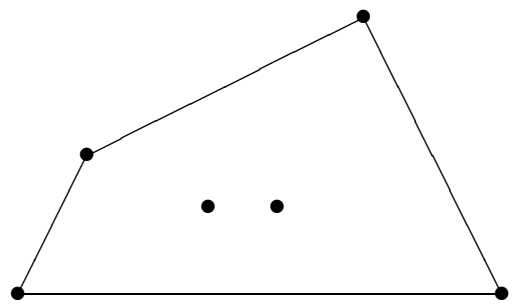

$\phi-\left(\mathcal{Q}_{1}(K)\right)^{2} \oplus S(K) b_{K}$

Figure 1

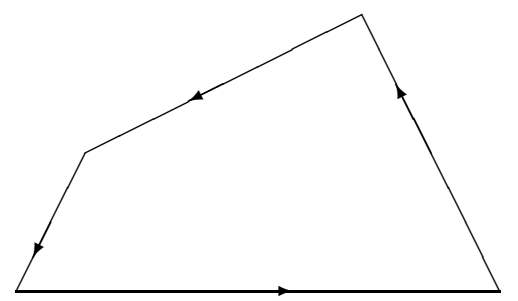

d.o.f. in $\mathbf{S}_{h}$

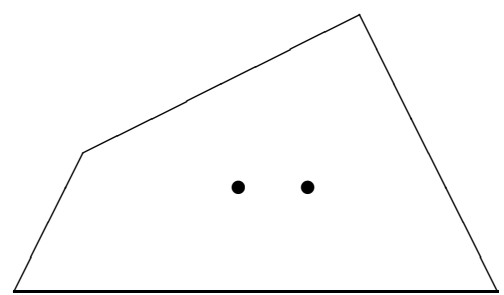

$\mathbf{q}-S(K)$

FIGURE 2

The new finite element method is to find $\left(\phi_{h}+\phi_{h}^{b}, w_{h}\right) \in \mathbf{H}_{h}^{+} \times W_{h}$ such that

$$
\begin{aligned}
& \frac{1}{1+\alpha} a\left(\phi_{h}, \psi_{h}\right)+\frac{\alpha}{1+\alpha} a\left(\phi_{h}+\phi_{h}^{b}, \psi_{h}+\psi_{h}^{b}\right) \\
& +\left(\mathbf{q}_{h}, \nabla v_{h}-\left(\Pi_{h} \psi_{h}+\frac{\alpha}{1+\alpha} \Pi_{h}^{0} \psi_{h}^{b}\right)\right)=\left(f, v_{h}\right) \\
& \quad \forall\left(\psi_{h}+\psi_{h}^{b}, v_{h}\right) \in \mathbf{H}_{h}^{+} \times W_{h}
\end{aligned}
$$


and

$$
\mathbf{q}_{h}=\lambda t^{-2}\left(\nabla w_{h}-\left(\Pi_{h} \phi_{h}+\frac{\alpha}{1+\alpha} \Pi_{h}^{0} \phi_{h}^{b}\right)\right) \in \Gamma_{h},
$$

where $\alpha>0$ is a constant which is specified in Theorem 3.1, and $\phi_{h} \in\left(W_{h}\right)^{2}$, $\phi_{h}^{b} \in \mathbf{B}_{h}$.

Theorem 3.1. If $0<\alpha<1$, then for all $\psi_{h}+\psi_{h}^{b} \in \mathbf{H}_{h}^{+}$, we have

$$
\frac{1}{1+\alpha} a\left(\psi_{h}, \psi_{h}\right)+\frac{\alpha}{1+\alpha} a\left(\psi_{h}+\psi_{h}^{b}, \psi_{h}+\psi_{h}^{b}\right) \geq C\left\{\left\|\psi_{h}\right\|_{1}^{2}+\left\|\psi_{h}^{b}\right\|_{1}^{2}\right\} .
$$

As a consequence, problem (3.6) and (3.7) has a unique solution $\left(\phi_{h}+\phi_{h}^{b}, w_{h}, \mathbf{q}_{h}\right)$.

Proof. In light of

$$
a\left(\psi_{h}+\psi_{h}^{b}, \psi_{h}+\psi_{h}^{b}\right) \geq \frac{1}{2} a\left(\psi_{h}^{b}, \psi_{h}^{b}\right)-a\left(\psi_{h}, \psi_{h}\right),
$$

put $0<\alpha<1$, we conclude that (3.8) holds.

Remark 3.1. In fact, any $\alpha$ in $\left(0, \frac{\epsilon}{1-\epsilon}\right),(1>\epsilon>0)$ can ensure (3.8); i.e., $\alpha$ may be any fixed positive constant. We may even take $\alpha=+\infty$. In fact, for all $\psi_{h} \neq \mathbf{0}$ and $\psi_{h}^{b} \neq \mathbf{0}$, let

$$
\sigma=\sup _{\mathbf{0} \neq \psi_{h} \in\left(W_{h}\right)^{2}, \mathbf{0} \neq \psi_{h}^{b} \in \mathbf{B}_{h}} \frac{\left|a\left(\psi_{h}, \psi_{h}^{b}\right)\right|}{a\left(\psi_{h}, \psi_{h}\right)^{1 / 2} a\left(\psi_{h}^{b}, \psi_{h}^{b}\right)^{1 / 2}} .
$$

We have $a\left(\psi_{h}+\psi_{h}^{b}, \psi_{h}+\psi_{h}^{b}\right) \geq(1-\sigma)\left\{a\left(\psi_{h}, \psi_{h}\right)+a\left(\psi_{h}^{b}, \psi_{h}^{b}\right)\right\}$. Only when some functions in $\left(W_{h}\right)^{2}$ and $\mathbf{B}_{h}$ satisfy $\psi_{h} \equiv \psi_{h}^{b}$ (scaled by a multiplicative constant), there hold $\sigma=1$; but, in this case, it is always true that $\psi_{h} \equiv \psi_{h}^{b} \equiv \mathbf{0}$. Of course, if $\psi_{h}=\mathbf{0}$ or $\psi_{h}^{b}=\mathbf{0}$, then (3.8) is trivial.

Note that in the case $\alpha=+\infty$, the method of (3.6) and (3.7) becomes

$$
\begin{aligned}
& a\left(\phi_{h}+\phi_{h}^{b}, \psi_{h}+\psi_{h}^{b}\right)+\left(\mathbf{q}_{h}, \nabla v_{h}-\left(\Pi_{h} \psi_{h}+\Pi_{h}^{0} \psi_{h}^{b}\right)\right)=\left(f, v_{h}\right) \\
& \forall\left(\psi_{h}+\psi_{h}^{b}, v_{h}\right) \in \mathbf{H}_{h}^{+} \times W_{h}
\end{aligned}
$$

and

$$
\mathbf{q}_{h}=\lambda t^{-2}\left(\nabla w_{h}-\left(\Pi_{h} \phi_{h}+\Pi_{h}^{0} \phi_{h}^{b}\right)\right) \in \Gamma_{h} .
$$

Obviously, if $\alpha=0$ and $\mathbf{B}_{h}$ is dropped, method (3.6) and (3.7) is none other than the MITC4 method with $\mathcal{T}_{h}$ composed of rectangles.

\section{ERror estimates}

In this section, we derive the $H^{1}$ error bounds for both transverse displacement and rotation.

Lemma 4.1. Let $\tilde{w} \in W_{h}, \tilde{\phi}+\tilde{\phi}^{b} \in \mathbf{H}_{h}^{+}$, and $\tilde{\mathbf{q}}=\lambda t^{-2}\left(\nabla \tilde{w}-\left(\Pi_{h} \tilde{\phi}+\frac{\alpha}{1+\alpha} \Pi_{h}^{0} \tilde{\phi}^{b}\right)\right)$ $\in \Gamma_{h}$. Then

$$
\begin{aligned}
& \left\|\tilde{\phi}-\phi_{h}\right\|_{1}+\left\|\tilde{\phi}^{b}+\tilde{\phi}-\left(\phi_{h}^{b}+\phi_{h}\right)\right\|_{1}+t\left\|\tilde{\mathbf{q}}-\mathbf{q}_{h}\right\|_{0} \\
& \leq C\left\{\|\tilde{\phi}-\phi\|_{1}+\|\tilde{\phi} b+\tilde{\phi}-\phi\|_{1}+t\|\tilde{\mathbf{q}}-\mathbf{q}\|_{0}+h\|\mathbf{q}\|_{0}\right\} .
\end{aligned}
$$


Proof. For all $\psi_{h}+\psi_{h}^{b} \in \mathbf{H}_{h}^{+}, v_{h} \in W_{h}$, from (2.1) to get

$$
\frac{1}{1+\alpha} a\left(\phi, \psi_{h}\right)+\frac{\alpha}{1+\alpha} a\left(\phi, \psi_{h}^{b}+\psi_{h}\right)+\left(\mathbf{q}, \nabla v_{h}-\left(\psi_{h}+\frac{\alpha}{1+\alpha} \psi_{h}^{b}\right)\right)=\left(f, v_{h}\right)
$$

which can be written as

$$
\begin{aligned}
& \frac{1}{1+\alpha} a\left(\phi, \psi_{h}\right)+\frac{\alpha}{1+\alpha} a\left(\phi, \psi_{h}^{b}+\psi_{h}\right)+\left(\mathbf{q}, \nabla v_{h}-\left(\Pi_{h} \psi_{h}+\frac{\alpha}{1+\alpha} \Pi_{h}^{0} \psi_{h}^{b}\right)\right) \\
& =\left(f, v_{h}\right)+\left(\mathbf{q}, \psi_{h}+\frac{\alpha}{1+\alpha} \psi_{h}^{b}-\left(\Pi_{h} \psi_{h}+\frac{\alpha}{1+\alpha} \Pi_{h}^{0} \psi_{h}^{b}\right)\right) .
\end{aligned}
$$

Subtracting (4.3) from (3.6), we have

$$
\begin{aligned}
& \frac{1}{1+\alpha} a\left(\phi-\phi_{h}, \psi_{h}\right)+\frac{\alpha}{1+\alpha} a\left(\phi-\left(\phi_{h}^{b}+\phi_{h}\right), \psi_{h}^{b}+\psi_{h}\right) \\
& +\left(\mathbf{q}-\mathbf{q}_{h}, \nabla v_{h}-\left(\Pi_{h} \psi_{h}+\frac{\alpha}{1+\alpha} \Pi_{h}^{0} \psi_{h}^{b}\right)\right) \\
& =\left(\mathbf{q}, \psi_{h}+\frac{\alpha}{1+\alpha} \psi_{h}^{b}-\left(\Pi_{h} \psi_{h}+\frac{\alpha}{1+\alpha} \Pi_{h}^{0} \psi_{h}^{b}\right)\right) .
\end{aligned}
$$

For any $\tilde{\phi}+\tilde{\phi}^{b} \in \mathbf{H}_{h}^{+}, \tilde{w} \in W_{h}$, let $\tilde{\mathbf{q}}=\lambda t^{-2}\left(\nabla \tilde{w}-\left(\Pi_{h} \tilde{\phi}+\frac{\alpha}{1+\alpha} \Pi_{h}^{0} \tilde{\phi}^{b}\right)\right) \in \Gamma_{h}$, from (4.4) to get

$$
\begin{aligned}
& \frac{1}{1+\alpha} a\left(\tilde{\phi}-\phi_{h}, \psi_{h}\right)+\frac{\alpha}{1+\alpha} a\left(\tilde{\phi}^{b}+\tilde{\phi}-\left(\phi_{h}^{b}+\phi_{h}\right), \psi_{h}^{b}+\psi_{h}\right) \\
& +\left(\tilde{\mathbf{q}}-\mathbf{q}_{h}, \nabla v_{h}-\left(\Pi_{h} \psi_{h}+\frac{\alpha}{1+\alpha} \Pi_{h}^{0} \psi_{h}^{b}\right)\right) \\
& =\frac{1}{1+\alpha} a\left(\tilde{\phi}-\phi, \psi_{h}\right)+\frac{\alpha}{1+\alpha} a\left(\tilde{\phi}^{b}+\tilde{\phi}-\phi, \psi_{h}^{b}+\psi_{h}\right) \\
& +\left(\tilde{\mathbf{q}}-\mathbf{q}, \nabla v_{h}-\left(\Pi_{h} \psi_{h}+\frac{\alpha}{1+\alpha} \Pi_{h}^{0} \psi_{h}^{b}\right)\right) \\
& +\left(\mathbf{q}, \psi_{h}+\frac{\alpha}{1+\alpha} \psi_{h}^{b}-\left(\Pi_{h} \psi_{h}+\frac{\alpha}{1+\alpha} \Pi_{h}^{0} \psi_{h}^{b}\right)\right) .
\end{aligned}
$$

In (4.5), let $\psi_{h}=\tilde{\phi}-\phi_{h} \in\left(W_{h}\right)^{2}, \psi_{h}^{b}=\tilde{\phi}^{b}-\phi_{h}^{b} \in \mathbf{B}_{h}, v_{h}=\tilde{w}-w_{h} \in W_{h}$, we have

$$
\tilde{\mathbf{q}}-\mathbf{q}_{h}=\lambda t^{-2}\left(\nabla v_{h}-\left(\Pi_{h} \psi_{h}+\frac{\alpha}{1+\alpha} \Pi_{h}^{0} \psi_{h}^{b}\right)\right)
$$

from which we have

$$
\begin{aligned}
& \frac{1}{1+\alpha} a\left(\tilde{\phi}-\phi_{h}, \tilde{\phi}-\phi_{h}\right)+\frac{\alpha}{1+\alpha} a\left(\tilde{\phi}^{b}+\tilde{\phi}-\left(\phi_{h}^{b}+\phi_{h}\right), \tilde{\phi}^{b}+\tilde{\phi}-\left(\phi_{h}^{b}+\phi_{h}\right)\right) \\
& +\frac{t^{2}}{\lambda}\left(\tilde{\mathbf{q}}-\mathbf{q}_{h}, \tilde{\mathbf{q}}-\mathbf{q}_{h}\right) \\
& =\frac{1}{1+\alpha} a\left(\tilde{\phi}-\phi, \tilde{\phi}-\phi_{h}\right)+\frac{\alpha}{1+\alpha} a\left(\tilde{\phi}^{b}+\tilde{\phi}-\phi, \tilde{\phi}^{b}+\tilde{\phi}-\left(\phi_{h}^{b}+\phi_{h}\right)\right) \\
& +\frac{t^{2}}{\lambda}\left(\tilde{\mathbf{q}}-\mathbf{q}, \tilde{\mathbf{q}}-\mathbf{q}_{h}\right) \\
& +\left(\mathbf{q}, \tilde{\phi}-\phi_{h}+\frac{\alpha}{1+\alpha}\left(\tilde{\phi}^{b}-\phi_{h}^{b}\right)-\left(\Pi_{h}\left(\tilde{\phi}-\phi_{h}\right)+\frac{\alpha}{1+\alpha} \Pi_{h}^{0}\left(\tilde{\phi}^{b}-\phi_{h}^{b}\right)\right)\right) .
\end{aligned}
$$


Therefore,

$$
\begin{aligned}
& \frac{1}{1+\alpha}\left\|\tilde{\phi}-\phi_{h}\right\|_{1}^{2}+\frac{\alpha}{1+\alpha}\left\|\tilde{\phi}^{b}+\tilde{\phi}-\left(\phi_{h}^{b}+\phi_{h}\right)\right\|_{1}^{2}+\frac{t^{2}}{\lambda}\left\|\tilde{\mathbf{q}}-\mathbf{q}_{h}\right\|_{0}^{2} \\
& \leq C\left\{\frac{1}{1+\alpha}\|\tilde{\phi}-\phi\|_{1}\left\|\tilde{\phi}-\phi_{h}\right\|_{1}\right. \\
& \left.\quad+\frac{\alpha}{1+\alpha}\left\|\tilde{\phi}^{b}+\tilde{\phi}-\phi\right\|_{1}\left\|\tilde{\phi}^{b}+\tilde{\phi}-\left(\phi_{h}^{b}+\phi_{h}\right)\right\|_{1}\right\} \\
& +\frac{t^{2}}{\lambda}\|\tilde{\mathbf{q}}-\mathbf{q}\|_{0}\left\|\tilde{\mathbf{q}}-\mathbf{q}_{h}\right\|_{0} \\
& +\|\mathbf{q}\|_{0}\left\|\tilde{\phi}-\phi_{h}-\Pi_{h}\left(\tilde{\phi}-\phi_{h}\right)+\frac{\alpha}{1+\alpha}\left(\tilde{\phi}^{b}-\phi_{h}^{b}-\Pi_{h}^{0}\left(\tilde{\phi}^{b}-\phi_{h}^{b}\right)\right)\right\|_{0}
\end{aligned}
$$

where

$$
\begin{aligned}
& \left\|\tilde{\phi}-\phi_{h}-\Pi_{h}\left(\tilde{\phi}-\phi_{h}\right)+\frac{\alpha}{1+\alpha}\left(\tilde{\phi}^{b}-\phi_{h}^{b}-\Pi_{h}^{0}\left(\tilde{\phi}^{b}-\phi_{h}^{b}\right)\right)\right\|_{0} \\
& \leq C h\left\{\left\|\tilde{\phi}-\phi_{h}\right\|_{1}+\left\|\tilde{\phi}^{b}-\phi_{h}^{b}\right\|_{1}\right\} \\
& \leq C h\left\{\left\|\tilde{\phi}-\phi_{h}\right\|_{1}+\left\|\tilde{\phi}^{b}+\tilde{\phi}-\left(\phi_{h}^{b}+\phi_{h}\right)\right\|_{1}\right\}
\end{aligned}
$$

from (4.8) and (4.9) to get (4.1).

On $W_{h}$, we introduce the standard Lagrangian interpolation operator $I_{h}$ as follows (cf. [18):

$$
\left\{\begin{array}{l}
I_{h}: w \in H_{0}^{1}(\Omega) \cap H^{2}(\Omega) \rightarrow I_{h} w \in W_{h} \\
I_{h} w(b)=w(b), \quad \text { for all vertex } b \\
\left\|I_{h} w-w\right\|_{0}+h\left|I_{h} w-w\right|_{1} \leq C h^{2}\|w\|_{2} .
\end{array}\right.
$$

On $\left(W_{h}\right)^{2}$, the corresponding interpolation operator is denoted by $\mathbf{I}_{h}=\left(I_{h}\right)^{2}$.

Lemma 4.2 (40], 33]). It holds that

$$
\nabla W_{h} \subset \mathbf{S}_{h} \subset \Gamma_{h} .
$$

Proof. This lemma was shown by [40], [33]. For the readers' convenience, here the proof is recalled.

For any $K \in \mathcal{T}_{h}$, with four vertices $\left(x_{i}, y_{i}\right)(1 \leq i \leq 4)$ and for any $v \in W_{h}$, let $v_{\mid K}=\sum_{i=1}^{4} v_{i}\left(N_{i} \circ F_{K}^{-1}\right)$, where $v_{i}, 1 \leq i \leq 4$, are nodal values of $v$ and $N_{i}$ $(1 \leq i \leq 4)$ are the corresponding bilinear polynomials on $\hat{K}$, i.e., the four local base functions of $\mathcal{Q}_{1}(K)$.

To show (4.11), we only need to show $\nabla\left(N_{i} \circ F_{K}^{-1}\right) \in S(K)$. Note that

$$
1=\sum_{i=1}^{4} N_{i} \circ F_{K}^{-1}, \quad x=\sum_{i=1}^{4} x_{i}\left(N_{i} \circ F_{K}^{-1}\right), \quad y=\sum_{i=1}^{4} y_{i}\left(N_{i} \circ F_{K}^{-1}\right),
$$


we have

$$
\left\{\begin{aligned}
\sum_{i=2}^{4} \nabla\left(N_{i} \circ F_{K}^{-1}\right) & =-\nabla\left(N_{1} \circ F_{K}^{-1}\right), \\
\sum_{i=2}^{4} x_{i} \nabla\left(N_{i} \circ F_{K}^{-1}\right) & =(1,0)^{T}-x_{1} \nabla\left(N_{1} \circ F_{K}^{-1}\right), \\
\sum_{i=2}^{4} y_{i} \nabla\left(N_{i} \circ F_{K}^{-1}\right) & =(0,1)^{T}-y_{1} \nabla\left(N_{1} \circ F_{K}^{-1}\right),
\end{aligned}\right.
$$

in light of the regularity of $\mathcal{T}_{h}$, we know that the coefficient matrix of (4.12) is nonsingular, and we solve (4.12) to get the conclusion.

Lemma 4.3. For any $w \in H_{0}^{1}(\Omega) \cap H^{2}(\Omega)$, the following property of commuting diagrams holds:

$$
\Pi_{h} \nabla w=\nabla I_{h} w .
$$

Proof. For any given edge $e$, let the two endpoints of $e$ be $A, B$. We then have (4.14)

$\int_{e} \Pi_{h} \nabla w \cdot \tau_{e}=\int_{e} \nabla w \cdot \tau_{e}=w(A)-w(B)=I_{h} w(A)-I_{h} w(B)=\int_{e} \nabla I_{h} w \cdot \tau_{e}$.

Note that $\nabla W_{h} \subset \mathbf{S}_{h}$ because of Lemma 4.2, we know that (4.13) is valid.

Lemma 4.4. Let $w \in H_{0}^{1}(\Omega) \cap H^{2}(\Omega), \phi \in\left(H_{0}^{1}(\Omega) \cap H^{2}(\Omega)\right)^{2}$. Let $\tilde{w}=I_{h} w \in$ $W_{h}, \tilde{\phi}=\mathbf{I}_{h} \phi \in\left(W_{h}\right)^{2}$, and let $\tilde{\phi}^{b} \in \mathbf{B}_{h}$ be defined by $\frac{\alpha}{1+\alpha} \Pi_{h}^{0} \tilde{\phi}^{b}=\Pi_{h}(\phi-\tilde{\phi})$. Then, for $\tilde{\mathbf{q}}=\lambda t^{-2}\left(\nabla \tilde{w}-\left(\Pi_{h} \tilde{\phi}+\frac{\alpha}{1+\alpha} \Pi_{h}^{0} \tilde{\phi}^{b}\right)\right) \in \Gamma_{h}$ and $\mathbf{q}=\lambda t^{-2}(\nabla w-\phi)$, it holds that

$$
\tilde{\mathbf{q}}=\Pi_{h} \mathbf{q}
$$

Proof. In fact,

$$
\begin{aligned}
\Pi_{h} \mathbf{q}=\lambda t^{-2}\left(\Pi_{h} \nabla w-\Pi_{h} \phi\right)= & \lambda t^{-2}\left(\nabla \tilde{w}-\Pi_{h} \phi\right) \\
= & \lambda t^{-2}\left(\nabla \tilde{w}-\left(\Pi_{h} \tilde{\phi}+\frac{\alpha}{1+\alpha} \Pi_{h}^{0} \tilde{\phi}^{b}\right)\right) \\
& +\lambda t^{-2}\left(\frac{\alpha}{1+\alpha} \Pi_{h}^{0} \tilde{\phi}^{b}-\Pi_{h}(\phi-\tilde{\phi})\right) \\
= & \tilde{\mathbf{q}}
\end{aligned}
$$

because of $\frac{\alpha}{1+\alpha} \Pi_{h}^{0} \tilde{\phi}^{b}=\Pi_{h}(\phi-\tilde{\phi})$.

Lemma 4.5. Let $\tilde{\phi}=\mathbf{I}_{h} \phi \in\left(W_{h}\right)^{2}$ and let $\tilde{\phi}^{b} \in \mathbf{B}_{h}$ be defined by $\frac{\alpha}{1+\alpha} \Pi_{h}^{0} \tilde{\phi}^{b}=$ $\Pi_{h}(\phi-\tilde{\phi})$. We have

$$
\left\|\phi-\left(\tilde{\phi}^{b}+\tilde{\phi}\right)\right\|_{1} \leq C h\|\phi\|_{2} .
$$

Proof. We first show

$$
\left\|\tilde{\phi}^{b}\right\|_{0, K} \leq C\left\|\Pi_{h}^{0} \tilde{\phi}^{b}\right\|_{0, K} .
$$

In fact, since $\tilde{\phi}_{\left.\right|_{K}}^{b} \in S(K) b_{K}$, let $\tilde{\phi}^{b}=\mathbf{s} b_{K}, \mathbf{s} \in S(K)$. Note that $b_{K} \leq C$,

$$
\left\|\tilde{\phi}^{b}\right\|_{0, K}^{2}=\int_{K} b_{K}^{2}|\mathbf{s}|^{2} \leq C \int_{K}|\mathbf{s}|^{2} b_{K}=C\left(\Pi_{h}^{0} \tilde{\phi}^{b}, \mathbf{s}\right)_{0, K} \leq C\left\|\Pi_{h}^{0} \tilde{\phi}^{b}\right\|_{0, K}\|\mathbf{s}\|_{0, K}
$$


and that $\|\mathbf{s}\|_{0, K}$ and $\left\|\mathbf{s} b_{K}\right\|_{0, K}=\left\|\tilde{\phi}^{b}\right\|_{0, K}$ are equivalent norms on $S(K)$. We obtain (4.18).

Next, we show

$$
\left\|\tilde{\phi}^{b}\right\|_{0, K} \leq C h_{K}^{2}\|\phi\|_{2, K}
$$

In fact,

$$
\begin{aligned}
\left\|\Pi_{h}^{0} \tilde{\phi}^{b}\right\|_{0, K} & =\frac{1+\alpha}{\alpha}\left\|\Pi_{h}(\phi-\tilde{\phi})\right\|_{0, K} \\
& \leq C\left\{\left\|\Pi_{h}(\phi-\tilde{\phi})-(\phi-\tilde{\phi})\right\|_{0, K}+\|\phi-\tilde{\phi} \mid\|_{0, K}\right\} \\
& \leq C h_{K}|\phi-\tilde{\phi}|_{1, K}+C h_{K}^{2}|\phi|_{2, K} \leq C h_{K}^{2}|\phi|_{2, K} .
\end{aligned}
$$

Hence, (4.20) is true. Finally,

$$
\left\|\phi-\left(\tilde{\phi}^{b}+\tilde{\phi}\right)\right\|_{1} \leq\|\phi-\tilde{\phi}\|_{1}+\left\|\tilde{\phi}^{b}\right\|_{1} \leq C h\|\phi\|_{2}
$$

where we have used $\|\phi-\tilde{\phi}\|_{1} \leq C h\|\phi\|_{2}$ and $\left\|\tilde{\phi}^{b}\right\|_{1} \leq C\left(\left\|\tilde{\phi}^{b}\right\|_{0}+\left|\tilde{\phi}^{b}\right|_{1}\right) \leq$ $C h^{-1}\left\|\tilde{\phi}^{b}\right\|_{0} \leq C h\|\phi\|_{2}$.

Theorem 4.1. Let $(\phi, w, \mathbf{q}) \in\left(H_{0}^{1}(\Omega)\right)^{2} \times H_{0}^{1}(\Omega) \times H_{0}$ (curl; $\left.\Omega\right)$ be the solution of (2.1) and (2.2), and let $\left(\phi_{h}+\phi_{h}^{b}, w_{h}, \mathbf{q}_{h}\right) \in \mathbf{H}_{h}^{+} \times W_{h} \times \Gamma_{h}$ be the solution of (3.6) and (3.7). Then

$$
\begin{aligned}
& \left\|\phi-\phi_{h}\right\|_{1}+\left\|w-w_{h}\right\|_{1}+t\left\|\mathbf{q}-\mathbf{q}_{h}\right\|_{0}+\left\|\phi-\left(\phi_{h}+\phi_{h}^{b}\right)\right\|_{1} \\
& \leq C h\left\{t\|\mathbf{q}\|_{1}+\|\phi\|_{2}+\|\mathbf{q}\|_{0}\right\} .
\end{aligned}
$$

Proof. Using the triangle-inequality, from Lemma 4.1 we have

$$
\begin{aligned}
& \left\|\phi-\phi_{h}\right\|_{1}+t\left\|\mathbf{q}-\mathbf{q}_{h}\right\|_{0}+\left\|\phi-\left(\phi_{h}+\phi_{h}^{b}\right)\right\|_{1} \\
& \leq\|\phi-\tilde{\phi}\|_{1}+t\|\tilde{\mathbf{q}}-\mathbf{q}\|_{0}+\left\|\phi-\left(\tilde{\phi}+\tilde{\phi}^{b}\right)\right\|_{1} \\
& +\left\|\tilde{\phi}-\phi_{h}\right\|_{1}+\left\|\tilde{\phi}+\tilde{\phi}^{b}-\left(\phi_{h}+\phi_{h}^{b}\right)\right\|_{1}+t\left\|\tilde{\mathbf{q}}-\mathbf{q}_{h}\right\|_{0} \\
& \leq C\left\{\|\tilde{\phi}-\phi\|_{1}+\left\|\tilde{\phi}^{b}+\tilde{\phi}-\phi\right\|_{1}+t\|\tilde{\mathbf{q}}-\mathbf{q}\|_{0}+h\|\mathbf{q}\|_{0}\right\}
\end{aligned}
$$

where, taking $\tilde{\phi}=\mathbf{I}_{h} \phi \in\left(W_{h}\right)^{2}$, from Lemmas 4.4 and 4.5 , we have

$$
\|\tilde{\phi}-\phi\|_{1}+t\|\tilde{\mathbf{q}}-\mathbf{q}\|_{0}+\left\|\tilde{\phi}^{b}+\tilde{\phi}-\phi\right\|_{1} \leq C h\left\{\|\phi\|_{2}+t\|\mathbf{q}\|_{1}\right\} .
$$

It follows from (4.23) and (4.22) that

$$
\left\|\phi-\left(\phi_{h}+\phi_{h}^{b}\right)\right\|_{1}+\left\|\phi-\phi_{h}\right\|_{1}+t\left\|\mathbf{q}-\mathbf{q}_{h}\right\|_{0} \leq C h\left\{t\|\mathbf{q}\|_{1}+\|\phi\|_{2}+\|\mathbf{q}\|_{0}\right\} .
$$

Note that

$$
\begin{aligned}
\nabla\left(w-w_{h}\right)= & \frac{t^{2}}{\lambda}\left(\mathbf{q}-\mathbf{q}_{h}\right)+\phi-\left(\Pi_{h} \phi_{h}+\frac{\alpha}{1+\alpha} \Pi_{h}^{0} \phi_{h}^{b}\right) \\
= & \frac{t^{2}}{\lambda}\left(\mathbf{q}-\mathbf{q}_{h}\right)+\phi-\Pi_{h} \phi \\
& +\Pi_{h}\left(\phi-\phi_{h}\right)-\left(\phi-\phi_{h}\right) \\
& +\phi-\phi_{h}-\frac{\alpha}{1+\alpha} \Pi_{h}^{0} \phi_{h}^{b} .
\end{aligned}
$$


We have

(4.26)

$$
\begin{aligned}
\left|w-w_{h}\right|_{1} \leq & \frac{t^{2}}{\lambda}\left\|\mathbf{q}-\mathbf{q}_{h}\right\|_{0}+\left\|\phi-\Pi_{h} \phi\right\|_{0} \\
& +\left\|\Pi_{h}\left(\phi-\phi_{h}\right)-\left(\phi-\phi_{h}\right)\right\|_{0}+\left\|\phi-\phi_{h}\right\|_{0}+\frac{\alpha}{1+\alpha}\left\|\Pi_{h}^{0} \phi_{h}^{b}\right\|_{0} \\
\leq & C h\left\{t\|\mathbf{q}\|_{1}+\|\phi\|_{2}+\|\mathbf{q}\|_{0}\right\}
\end{aligned}
$$

where we have used $\left\|\Pi_{h}^{0} \phi_{h}^{b}\right\|_{0} \leq\left\|\phi_{h}^{b}\right\|_{0} \leq C h\left\{t\|\mathbf{q}\|_{1}+\|\phi\|_{2}+\|\mathbf{q}\|_{0}\right\}$ because of (4.24).

Remark 4.1. Note that when $\Omega$ is a convex polygon, the solution of (2.1) and (2.2) satisfies the prior regularity estimation (cf. [30], 23], [8])

$$
\|\phi\|_{2}+\|\mathbf{q}\|_{0}+t\|\mathbf{q}\|_{1} \leq C\|f\|_{0} .
$$

From Theorem 4.1 we have

$$
\left\|\phi-\phi_{h}\right\|_{1}+\left\|w-w_{h}\right\|_{1}+t\left\|\mathbf{q}-\mathbf{q}_{h}\right\|_{0}+\left\|\phi-\left(\phi_{h}+\phi_{h}^{b}\right)\right\|_{1} \leq C h\|f\|_{0} .
$$

Therefore, the method (3.6) and (3.7) is locking-free.

\section{5. $L^{2}$ ERROR BOUND}

This section is concerned with the $L^{2}$ error bounds for both transverse displacement and rotation. The domain $\Omega$ is a convex polygon.

Introduce

$$
H(\operatorname{div} ; \Omega)=\left\{\mathbf{s} \in\left(L^{2}(\Omega)\right)^{2} ; \operatorname{div} \mathbf{s} \in L^{2}(\Omega)\right\}
$$

with the norm $\|\cdot\|_{H(\operatorname{div} ; \Omega)}^{2}=\|\cdot\|_{0}^{2}+\|\operatorname{div} \cdot\|_{0}^{2}$.

Lemma 5.1 (cf. [8], 34]). For every $\mathbf{q} \in\left(L^{2}(\Omega)\right)^{2}$, the following Helmholtzdecomposition holds:

$$
\mathbf{q}=\nabla u+\operatorname{curl} p, \quad u \in H_{0}^{1}(\Omega), p \in H^{1}(\Omega) / \Re .
$$

Lemma 5.2. Let $\mathcal{Q}_{2}(K), K \in \mathcal{T}_{h}$, be the space of isoparametric biquadratic polynomials, and let $\varphi_{i}, 1 \leq i \leq 4$, be the four base functions of $\mathcal{Q}_{2}(K)$ corresponding to the four midpoints of $\partial \bar{K}=\left\{e_{i}, 1 \leq i \leq 4\right\}$. Then, for any given $\psi_{h} \in\left(W_{h}\right)^{2}$, there exists a $v_{0} \in H_{0}^{1}(\Omega)$ such that

$$
\begin{gathered}
v_{\left.0\right|_{K}} \in \operatorname{span}\left\{\varphi_{i}, 1 \leq i \leq 4\right\}, \\
\left(\psi_{h}-\nabla v_{0}\right) \cdot \tau_{e_{i}}=\frac{1}{\left|e_{i}\right|} \int_{e_{i}} \psi_{h} \cdot \tau_{e_{i}}, \quad 1 \leq i \leq 4, \\
\left|v_{0}\right|_{1, K} \leq C h_{K}\left|\psi_{h}\right|_{1, K}, \\
\left\|v_{0}\right\|_{0} \leq C h\left|v_{0}\right|_{1} \leq C h^{2}\left|\psi_{h}\right|_{1} .
\end{gathered}
$$

Proof. Formulas (5.2)-(5.4) can be obtained through the argument in [38, [39]. Regarding (5.5), since $v_{0}$ vanishes on $\partial \Omega$ and vanishes at all the nodes of the triangulation and $v_{\left.0\right|_{K}} \in \mathcal{Q}_{2}(K)$, a standard scaling argument on each element $K$ yields $\left\|v_{0}\right\|_{0} \leq C h\left|v_{0}\right|_{1}$.

Corollary 5.1. Let $\Pi_{h} \psi_{h} \in \mathbf{S}_{h}$ be the interpolant to $\psi_{h} \in\left(W_{h}\right)^{2}$. Then

$$
\psi_{h}-\Pi_{h} \psi_{h}-\nabla v_{0} \in H_{0}(\operatorname{curl} ; K)
$$


where

$$
H_{0}(\operatorname{curl} ; K)=\left\{\mathbf{s} \in\left(L^{2}(K)\right)^{2} ; \operatorname{curl} \mathbf{s} \in L^{2}(K), \mathbf{s} \cdot \tau_{\left.\right|_{\partial K}}=0\right\}
$$

with $\tau$ the tangent to $\partial K$, and $v_{0}$ is constructed as in Lemma 5.2.

The following result is well known (cf. [34, [18]).

Lemma 5.3. For any given $v \in H_{0}^{1}(K), K \in \mathcal{T}_{h}$. Then

$$
\|v\|_{0, K} \leq C h_{K}|v|_{1, K} .
$$

Theorem 5.1. For any $\psi_{h} \in\left(W_{h}\right)^{2}$, let $\Pi_{h} \psi_{h} \in \mathbf{S}_{h}$ be the interpolant. Then, the following Helmholtz-decomposition holds:

$$
\psi_{h}-\Pi_{h} \psi_{h}=\nabla r+\operatorname{curl}_{h} s,
$$

where curl $_{h}$ is the curl operator element-by-element, $r \in H_{0}^{1}(\Omega), s \in H^{1}(K) / \Re$, $\forall K \in \mathcal{T}_{h}$, and we have

$$
\begin{gathered}
\|r\|_{0} \leq C h^{2}\left|\psi_{h}\right|_{1}, \\
|s|_{1, h} \leq C h^{2}\left|\operatorname{curl} \psi_{h}\right|_{1, h},
\end{gathered}
$$

where $|s|_{1, h}=\left(\sum_{K \in \mathcal{T}_{h}} \| \text { curls } \|_{0, K}^{2}\right)^{1 / 2}$.

Proof. As in Lemma 5.2 and Corollary 5.1, we can find a $v_{0} \in H_{0}^{1}(\Omega)$ such that

$$
\begin{aligned}
\psi_{h}-\Pi_{h} \psi_{h}-\nabla v_{0} & \in H_{0}(\operatorname{curl} ; K), \quad \forall K \in \mathcal{T}_{h}, \\
\left\|v_{0}\right\|_{0} & \leq C h^{2}\left|\psi_{h}\right|_{1} .
\end{aligned}
$$

On each $K \in \mathcal{T}_{h}$, let the Helmholtz-decomposition for $\psi_{h}-\Pi_{h} \psi_{h}-\nabla v_{0}$ be

$$
\psi_{h}-\Pi_{h} \psi_{h}-\nabla v_{0}=\nabla r_{K}+\operatorname{curl} s_{K}, \quad r_{K} \in H_{0}^{1}(K), s_{K} \in H^{1}(K) / \Re .
$$

Note that $\left(\nabla r_{K}, \operatorname{curl} s_{K}\right)_{0, K}=0$ and $\psi_{h}-\Pi_{h} \psi_{h}-\nabla v_{0} \in H_{0}(\operatorname{curl} ; K)$ and the property of commuting diagrams (2.14) and Lemma 5.2 hold, from (5.13) we have

$$
\begin{aligned}
\left\|\operatorname{curl} s_{K}\right\|_{0, K}^{2} & =\left(\psi-\Pi_{h} \psi_{h}-\nabla v_{0}, \operatorname{curl} s_{K}\right)_{0, K} \\
& =\left(\operatorname{curl}\left(\psi_{h}-\Pi_{h} \psi_{h}-\nabla v_{0}\right), s_{K}\right)_{0, K} \\
& =\left(\operatorname{curl} \psi_{h}-P_{h} \operatorname{curl} \psi_{h}, s_{K}\right)_{0, K} \\
& =\left(\operatorname{curl} \psi_{h}-P_{h} \operatorname{curl} \psi_{h}, s_{K}-P_{h} s_{K}\right)_{0, K} \\
& \leq C h_{K}^{2}\left|\operatorname{curl} \psi_{h}\right|_{1, K}\left|s_{K}\right|_{1, K},
\end{aligned}
$$

that is,

$$
\begin{gathered}
\left\|\operatorname{curl} s_{K}\right\|_{0, K} \leq C h_{K}^{2}\left|\operatorname{curl} \psi_{h}\right|_{1, K}, \\
\left|r_{K}\right|_{1, K} \leq\left\{\left\|\psi_{h}-\Pi_{h} \psi_{h}\right\|_{0, K}+\left\|\nabla v_{0}\right\|_{0, K}\right\} \leq C h_{K}\left|\psi_{h}\right|_{1, K} .
\end{gathered}
$$

It follows from Lemma 5.3 that

$$
\|\left. r_{K}\right|_{0, K} \leq C h_{K}\left|r_{K}\right|_{1, K} \leq C h_{K}^{2}\left|\psi_{h}\right|_{1, K} .
$$

Define $r_{0} \in H_{0}^{1}(\Omega)$ and $s \in L^{2}(\Omega)$ as follows:

$$
r_{\left.0\right|_{K}}=r_{K}, \quad s_{\left.\right|_{K}}=s_{K}, \quad \forall K \in \mathcal{T}_{h} .
$$

Then letting $r=v_{0}+r_{0} \in H_{0}^{1}(\Omega)$, we have the conclusion. 
Theorem 5.2. Let $\mathbf{q} \in H(\operatorname{div} ; \Omega), \beta \in\left(H^{2}(\Omega) \cap H_{0}^{1}(\Omega)\right)^{2}$, and $\tilde{\beta}=\mathbf{I}_{h} \beta \in\left(W_{h}\right)^{2}$. Then

$$
\left(\mathbf{q}, \tilde{\beta}-\Pi_{h} \tilde{\beta}\right) \leq C h^{2}\|\mathbf{q}\|_{H(\operatorname{div} ; \Omega)}\|\beta\|_{2} .
$$

Proof. From Lemma 5.1, the Helmholtz-decomposition for $\mathbf{q}$ is as follows:

$$
\mathbf{q}=\nabla u+\operatorname{curl} p, \quad u \in H_{0}^{1}(\Omega), \quad p \in H^{1}(\Omega) / \Re .
$$

Note that $\Omega$ is a convex polygon and $\mathbf{q} \in H(\operatorname{div} ; \Omega)$, we have $u \in H^{2}(\Omega)$.

From Theorem 5.1, $\tilde{\beta}-\Pi_{h} \tilde{\beta}$ can be written as

$$
\tilde{\beta}-\Pi_{h} \tilde{\beta}=\nabla r+\operatorname{curl}_{h} s, \quad r \in H_{0}^{1}(\Omega), \quad s \in H^{1}(K) / \Re, \forall K \in \mathcal{T}_{h}
$$

where

$$
\begin{gathered}
\|r\|_{0} \leq C h^{2}|\tilde{\beta}|_{1} \leq C h^{2}\|\beta\|_{2}, \\
|s|_{1, h} \leq C h^{2}|\operatorname{curl} \tilde{\beta}|_{1, h} \leq C h^{2}\|\beta\|_{2} .
\end{gathered}
$$

Note that

$$
\left(\mathbf{q}, \tilde{\beta}-\Pi_{h} \tilde{\beta}\right)=(\nabla u, \nabla r)+\left(\nabla u, \operatorname{curl}_{h} s\right)+\left(\operatorname{curl} p, \tilde{\beta}-\Pi_{h} \tilde{\beta}\right),
$$

from (5.22) and (5.23), we have

$$
\begin{gathered}
(\nabla u, \nabla r)=-(\Delta u, r) \leq\|\operatorname{div} \mathbf{q}\|_{0}\|r\|_{0} \leq C h^{2}\|\operatorname{div} \mathbf{q}\|_{0}\|\beta\|_{2}, \\
\left(\nabla u, \operatorname{curl}_{h} s\right) \leq|u|_{1}|s|_{1, h} \leq C h^{2}|u|_{1}\|\beta\|_{2} .
\end{gathered}
$$

On the other hand, in light of

$$
\left(\operatorname{curl} p, \tilde{\beta}-\Pi_{h} \tilde{\beta}\right)=\left(\operatorname{curl} p, \beta-\Pi_{h} \beta\right)+\left(\operatorname{curl} p, \Pi_{h}(\beta-\tilde{\beta})-(\beta-\tilde{\beta})\right),
$$

using the property of the commuting of diagrams (2.14), we have

$$
\begin{aligned}
&\left(\operatorname{curl} p, \beta-\Pi_{h} \beta\right)=\left(p, \operatorname{curl}\left(\beta-\Pi_{h} \beta\right)\right)=\left(p, \operatorname{curl} \beta-P_{h} \operatorname{curl} \beta\right) \\
&=\left(p-P_{h} p, \operatorname{curl} \beta-P_{h} \operatorname{curl} \beta\right) \\
& \leq C h^{2}\|p\|_{1}\|\beta\|_{2}, \\
& \text { 29) } \quad\left(\operatorname{curl} p, \Pi_{h}(\beta-\tilde{\beta})-(\beta-\tilde{\beta})\right) \leq C h\|p\|_{1}\|\beta-\tilde{\beta}\|_{1} \leq C h^{2}\|p\|_{1}\|\beta\|_{2} .
\end{aligned}
$$

Summarizing from (5.24) to (5.29), we get (5.19).

Corollary 5.2. For any $\mathbf{q} \in H(\operatorname{div} ; \Omega), \beta \in\left(H_{0}^{1}(\Omega) \cap H^{2}(\Omega)\right)^{2}$ and $\psi_{h} \in \mathbf{S}_{h}$, we have

$$
\begin{gathered}
\left(\mathbf{q}, \beta-\Pi_{h} \beta\right) \leq C h^{2}\|\mathbf{q}\|_{H(\operatorname{div} ; \Omega)}\|\beta\|_{2}, \\
\left(\mathbf{q}, \psi_{h}-\Pi_{h} \psi_{h}\right) \leq C h\|\mathbf{q}\|_{H(\operatorname{div} ; \Omega)}\left|\beta-\psi_{h}\right|_{1}+C h^{2}\|\mathbf{q}\|_{H(\operatorname{div} ; \Omega)}\|\beta\|_{2} .
\end{gathered}
$$

Proof. Let $\tilde{\beta}=\mathbf{I}_{h} \beta \in\left(W_{h}\right)^{2}$. Note that

$$
\left(\mathbf{q}, \beta-\Pi_{h} \beta\right)=\left(\mathbf{q}, \tilde{\beta}-\Pi_{h} \tilde{\beta}\right)+\left(\mathbf{q}, \Pi_{h}(\tilde{\beta}-\beta)-(\tilde{\beta}-\beta)\right),
$$

in light of the standard interpolation properties of $\mathbf{I}_{h}$ and $\Pi_{h}$ and Theorem 5.2, we conclude that (5.30) holds. Note that

$$
\left(\mathbf{q}, \psi_{h}-\Pi_{h} \psi_{h}\right)=\left(\psi_{h}-\beta-\Pi_{h}\left(\psi_{h}-\beta\right), \mathbf{q}\right)+\left(\beta-\Pi_{h} \beta, \mathbf{q}\right) .
$$

We immediately have (5.31). 
Remark 5.1. The key point to showing the $L^{2}$ error bounds in Theorem 5.3 below is Theorem 5.2. In fact, the main difficulty in deriving the $L^{2}$ error bound for the method in which the reduction operator involves $\Pi_{h}$ is how to bound the inconsistency term (5.19) (or (5.30), (5.31)). For the MITC3 element, a different approach to showing (5.19) is given by [37. Of course, if $\mathbf{S}_{h}$ includes the space of higher-order polynomials (at least linear), (5.19) is trivial (cf. [15], 7], 35]). However, the approaches in [37, [15, [7, 35] are not applicable here.

Theorem 5.3. Let $\Omega$ be a convex polygon. Let $(w, \phi)$ and $\left(w_{h}, \phi_{h}+\phi_{h}^{b}\right)$ be the exact and approximate solutions, respectively. Then

$$
\left\|w-w_{h}\right\|_{0}+\left\|\phi-\phi_{h}\right\|_{0}+\left\|\phi_{h}^{b}\right\|_{0} \leq C h^{2}\|f\|_{0} .
$$

Proof. Consider the dual problem: Find $(\beta, z) \in\left(H_{0}^{1}(\Omega)\right)^{2} \times H_{0}^{1}(\Omega)$ such that

$$
a(\beta, \psi)+(\nabla v-\psi, \gamma)=\left(\psi, \phi-\left(\phi_{h}+\frac{\alpha}{1+\alpha} \phi_{h}^{b}\right)\right)+\left(w-w_{h}, v\right)
$$

and

$$
\gamma=\lambda t^{-2}(\nabla z-\beta) \in H_{0}(\operatorname{curl} ; \Omega)
$$

for all $(\psi, v) \in\left(H_{0}^{1}(\Omega)\right)^{2} \times H_{0}^{1}(\Omega)$.

From [30, 23], [8], 9] we know that

$$
\|\beta\|_{2}+\|z\|_{2}+t\|\gamma\|_{1}+\|\gamma\|_{H(\operatorname{div} ; \Omega)} \leq C\left\{\left\|\phi-\left(\phi_{h}+\frac{\alpha}{1+\alpha} \phi_{h}^{b}\right)\right\|_{0}+\left\|w-w_{h}\right\|_{0}\right\} .
$$

Take $\psi=\phi-\left(\phi_{h}+\frac{\alpha}{1+\alpha} \phi_{h}^{b}\right), v=w-w_{h}$, and $\left(\tilde{\beta}+\tilde{\beta}^{b}, \tilde{z}\right) \in \mathbf{H}_{h}^{+} \times W_{h}, \tilde{\gamma}=$ $\lambda t^{-2}\left(\nabla \tilde{z}-\left(\Pi_{h} \tilde{\beta}+\frac{\alpha}{1+\alpha} \Pi_{h}^{0} \tilde{\beta}^{b}\right)\right) \in \Gamma_{h}$. In light of $\mathbf{q}=\lambda t^{-2}(\nabla w-\phi)$ and $\mathbf{q}_{h}=$ $\lambda t^{-2}\left(\nabla w_{h}-\left(\Pi_{h} \phi_{h}+\frac{\alpha}{1+\alpha} \Pi_{h}^{0} \phi_{h}^{b}\right)\right)$, we have

$$
\begin{aligned}
& \left\|\phi-\left(\phi_{h}+\frac{\alpha}{1+\alpha} \phi_{h}^{b}\right)\right\|_{0}^{2}+\left\|w-w_{h}\right\|_{0}^{2} \\
& =a\left(\beta, \phi-\left(\phi_{h}+\frac{\alpha}{1+\alpha} \phi_{h}^{b}\right)\right)+\frac{t^{2}}{\lambda}\left(\mathbf{q}-\mathbf{q}_{h}, \gamma\right) \\
& +\left(\phi_{h}+\frac{\alpha}{1+\alpha} \phi_{h}^{b}-\left(\Pi_{h} \phi_{h}+\frac{\alpha}{1+\alpha} \Pi_{h}^{0} \phi_{h}^{b}\right), \gamma\right) \\
& =\frac{1}{1+\alpha} a\left(\beta-\tilde{\beta}, \phi-\phi_{h}\right)+\frac{\alpha}{1+\alpha} a\left(\beta-\left(\tilde{\beta}+\tilde{\beta}^{b}\right), \phi-\left(\phi_{h}+\phi_{h}^{b}\right)\right) \\
& +\frac{t^{2}}{\lambda}\left(\mathbf{q}-\mathbf{q}_{h}, \gamma-\tilde{\gamma}\right)+\left(\mathbf{q}, \tilde{\beta}+\frac{\alpha}{1+\alpha} \tilde{\beta}^{b}-\left(\Pi_{h} \tilde{\beta}+\frac{\alpha}{1+\alpha} \Pi_{h}^{0} \tilde{\beta}_{h}^{b}\right)\right) \\
& +\left(\phi_{h}+\frac{\alpha}{1+\alpha} \phi_{h}^{b}-\left(\Pi_{h} \phi_{h}+\frac{\alpha}{1+\alpha} \Pi_{h}^{0} \phi_{h}^{b}\right), \gamma\right),
\end{aligned}
$$

where we have used (4.4) with $v_{h}=\tilde{z}$ and $\psi_{h}+\psi_{h}^{b}=\tilde{\beta}+\tilde{\beta}^{b}$.

Equation (5.38) indicates that we only need to estimate the last two terms in (5.38). To do so, let $\tilde{z}=I_{h} z \in W_{h}, \tilde{\beta}=\mathbf{I}_{h} \beta \in\left(W_{h}\right)^{2}$, and $\tilde{\beta}^{b}$ be defined by $\frac{\alpha}{1+\alpha} \Pi_{h}^{0} \tilde{\beta}^{b}=\Pi_{h}(\beta-\tilde{\beta})$. From Lemmas 4.4 and 4.5 , we have

$$
\tilde{\gamma}=\Pi_{h} \gamma, \quad\|\tilde{\gamma}-\gamma\|_{0} \leq C h|\gamma|_{1}, \quad\left\|\tilde{\beta}^{b}\right\|_{1} \leq C h\|\beta\|_{2} .
$$


In light of Corollary 5.2 and Theorem 4.1, we have

$$
\begin{aligned}
& \left|\left(\phi_{h}+\frac{\alpha}{1+\alpha} \phi_{h}^{b}-\left(\Pi_{h} \phi_{h}+\frac{\alpha}{1+\alpha} \Pi_{h}^{0} \phi_{h}^{b}\right), \gamma\right)\right| \\
& \leq\left|\left(\phi_{h}-\Pi_{h} \phi_{h}, \gamma\right)\right|+C h\|\gamma\|_{0}\left|\phi_{h}^{b}\right|_{1} \\
& \leq C h\left|\phi-\phi_{h}\right|_{1}\|\gamma\|_{0}+C h^{2}\|\gamma\|\left\|_{H(\operatorname{div} ; \Omega)}\right\| \phi\left\|_{2}+C h^{2}\right\| f\left\|_{0}\right\| \gamma \|_{0} \\
& \leq C h^{2}\|f\|_{0}\|\gamma\|_{H(\operatorname{div} ; \Omega)}, \\
& \quad\left|\left(\mathbf{q}, \tilde{\beta}+\frac{\alpha}{1+\alpha} \tilde{\beta}^{b}-\left(\Pi_{h} \tilde{\beta}+\frac{\alpha}{1+\alpha} \Pi_{h}^{0} \tilde{\beta}_{h}^{b}\right)\right)\right| \\
& \leq\left|\left(\mathbf{q}, \tilde{\beta}-\Pi_{h} \tilde{\beta}\right)\right|+C h\|\mathbf{q}\|_{0}\left|\tilde{\beta}^{b}\right|_{1} \leq C h^{2}\|\mathbf{q}\|_{H(\operatorname{div} ; \Omega)}\|\beta\|_{2} .
\end{aligned}
$$

Therefore, from (5.38)-(5.41) and (5.37), we conclude that

$$
\left\|\phi-\left(\phi_{h}+\frac{\alpha}{1+\alpha} \phi_{h}^{b}\right)\right\|_{0}+\left\|w-w_{h}\right\|_{0} \leq C h^{2}\|f\|_{0} .
$$

Finally, in light of Lemma 5.3 and Theorem 4.1, we know that

$$
\left\|\phi_{h}^{b}\right\|_{0} \leq C h\left|\phi_{h}^{b}\right|_{1} \leq C h^{2}\|f\|_{0},
$$

which completes the proof.

\section{ACKNOWLEDGMENTS}

The authors wish to thank the anonymous referees for many constructive comments that improved the paper greatly.

\section{REFERENCES}

[1] K.J. Bathe and E.N. Dvorkin, A four-node plate bending element based on Mindlin-Reissner theory and a mixed interpolation, Internat. J. Numer. Methods Engrg., 21(1985), pp. 367-383.

[2] K.J. Bathe and F. Brezzi, On the convergence of a four-node plate bending element based on Mindlin-Reissner plate theory and a mixed interpolation, MAFELAP, V, J.R. Whiteman, ed., London, (1985), pp. 491-503. MR 87f:65125

[3] R. Durán and E. Liberman, On mixed finite element methods for the Reissner-Mindlin plate model, Math. Comp., 58(1992), pp. 561-573. MR 92f:65135

[4] M. Lyly, R. Stenberg and T. Vihinen, A stable bilinear element for the Reissner-Mindlin plate model, Comput. Methods Appl. Mech. Engrg, 110(1993), pp. 343-357. MR 94k:73072

[5] R.S. Falk and Tong Tu, Locking-free finite elements for the Reissner-Mindlin plate, Math. Comp., 69(1999), pp. 911-928. MR 2000j:74088

[6] T.-X. Zhou, The partial projection method in the finite element discretization of the ReissnerMindlin plate, J. Comput. Math, 13(1995), pp. 172-191. MR 96m:73052

[7] F. Brezzi, M. Fortin and R. Stenberg, Error analysis of mixed-interpolated elements for Reissner-Mindlin plates, Math. Models and Methods in Appl. Sci, 1(1991), pp. 125-151. MR 92e:73030

[8] F. Brezzi and M. Fortin, Mixed and Hybrid Finite Element Methods, Springer-Verlag, 1991. MR 92d:65187

[9] D.N. Arnold and R.S. Falk, The boundary layer for the Reissner-Mindlin plate model, SIAM. J. Math. Anal., 21(1990), pp. 281-312. MR 91c:73053

[10] H.-Y. Duan and G.-P. Liang, Analysis of some stabilized low-order mixed finite element methods for Reissner-Mindlin plates, Comput. Methods Appl. Mech. Engrg., 191(2001), pp. 157-179. MR 2002h:74071

[11] R. Stenberg, A new finite element formulation for the plate bending problem, in: Asymptotic Methods for Elastic Structures, Eds: Ciarlet/Trabucho/Viano, Walter de Gruyter \& Co., Berlin, 1995, pp. 209-221. MR 96k:73084

[12] J. Pitkäranta and M. Suri, Design principles and error analysis for reduced-shear platebending finite elements, Numer. Math., 75(1996), pp. 223-266. MR 98c:73078 
[13] J. Pitkäranta, Analysis of some low-order finite element schemes for Mindlin-Reissner and Kirchhoff plates, Numer. Math., 53(1988), pp. 237-254. MR 89f:65126

[14] P. Destuynder, Mathematical Analysis of Thin Shell Problems, Masson, Paris, 1991.

[15] P. Peisker and D. Braess, Uniform convergence of mixed interpolated elements for ReissnerMindlin plates, M² AN Math. Model. Numer. Anal., 26(1992), pp. 557-574. MR 93j:73070

[16] F. Auricchio and C. Lovadina, Analysis of kinematic linked interpolation methods for Reissner-Mindlin plate problems, Comput. Methods Appl. Mech. Engrg., 190(2001), pp. 24652482. MR 2002b:74061

[17] R. Durán, A. Ghioldi and N. Wolanski, A finite element method for the Reissner-Mindlin plate model, SIAM J. Numer. Anal., 28(1991), pp. 1004-1014. MR 92f:73046

[18] P.G. Ciarlet, The Finite Element Method for Elliptic Problems, North-Holland, Amsterdam, 1978. MR 58:25001

[19] K.-J. Bathe, Finite Element Procedures, Prentice Hall, 1996. MR 87j:65144

[20] A. Iosilevich, K.-J. Bathe and F. Brezzi, Numerical Inf-Sup analysis of MITC plate bending elements, in: Plates and Shells, CRM Proceedings and Lecture Notes, M. Fortin (ed.), AMS, Providence, Vol. 21, 1999, pp. 225-242. MR 2000d:74076

[21] Z.-C. Shi, A convergence condition for the quadrilateral Wilson element, Numer. Math., 44(1984), pp. 349-361. MR 86d:65151

[22] M. Lyly and R. Stenberg, Stabilized MITC plate bending elements, in: M. Papadrakakis and B.H.V. Topping, eds., Advances in Finite Element Techniques, CIVIL-COMP Ltd, Edinburgh, Scotland, 1994, pp. 11-16.

[23] F. Brezzi and M. Fortin, Numerical approximation of Reissner-Mindlin plates, Math. Comp., 47(1986), pp. 151-158. MR 87g:73057

[24] S.L. Weissman and R.L. Taylor, Resultant fields for mixed plate bending elements, Comput. Methods Appl. Mech. Engrg., 79(1990), pp. 321-355. MR 91a:73071

[25] O.C. Zienkiewicz, Z. Xu, L.F. Zeng, A. Samuelsson and N.E. Wiberg, Linked interpolation for Reissner-Mindlin plate elements: Part I-A simple quadrilateral; Part III-An alternative quadrilateral, Int. J. Numer. Methods Engrg., 36(1993), pp. 3043-3056; 37(1994), pp. 14371443.

[26] R. L. Taylor and F. Auricchio, Linked interpolation for Reissner-Mindlin plate elements: Part II-a simple triangle, Int. J. Numer Methods Engrg., 36(1993), pp. 3057-3066.

[27] F. Auricchio and C. Lovadina, Partial selected reduced integration schemes and kinematically linked interpolations for plate bending problems, Math. Model. Methods Appl. Sci., 9(1999), pp. 693-722. MR 2001c:74080

[28] C. Chapelle and R. Stenberg, An optimal low-order lock-free finite element method for Reissner- Mindlin plates, Math. Models and Methods in Applied Sciences, 8(1998), pp. 407430. MR 99d:73088

[29] F. Brezzi, K.J. Bathe and M. Fortin, Mixed-interpolated elements for Mindlin-Reissner plate, Internat. J. Numer. Methods Engrg., 28(1989), pp. 1787-1801. MR 90g:73090

[30] D.N. Arnold and R.S. Falk, A uniformly accurate finite element method for the ReissnerMindlin plates, SIAM J. Numer. Anal., 26(1989), pp. 1276-1290. MR 91c:65068

[31] J. Pitkäranta and R. Stenberg, Error bounds for the approximation of Stokes problem with bilinear/constant elements on irregular quadrilateral meshes, in: J. R. Whiteman, ed., The Mathematics of Finite Elements and Applications, V. MAFELAP, Academic Press, NewYork, 1985, pp. 325-334.

[32] K. J. Bathe and F. Brezzi, A simplified analysis of two plate bending elements-the MITC4 and MITC9 elements, in: G.N. Pande and J. Middleton, eds., NUMETA 87, Vol. 1, Numerical Techniques for Engineering Analysis and Design, Martinus Nijhoff, Amsterdam, 1987.

[33] H.-Y Duan and G.-P. Liang, Nonconforming elements in least-squares mixed finite element methods, Math. Comp. 73(2004), pp. 1-18.

[34] V. Girault and P.A. Raviart, Finite Element Methods for Navier-Stokes Equations, SpringerVerlag, Berlin, 1986. MR 88b:65129

[35] M. Lyly and R. Stenberg, Stabilized finite element methods for Reissner-Mindlin plates (in: the web page of the second author)

[36] J. P. Wang and T. Mathew, Mixed finite element methods over quadrilaterals, in: Proceedings of 3rd Internat. Conference on Advances in Numerical Methods and Applications, J.T. Dimov, B. Sendov, and P. Vassilevski, eds., World Scientific, River Edge, NJ, 1994, pp. 203-214. 
[37] R. Durán, L. Hervella-Nieto, E. Liberman, R. Rodriguez and J. Solomin, Approximation of the vibration modes of a plate by Reissner-Mindlin equation, Math. Comp., 68(1999), pp. 1447-1463. MR 99m:73045

[38] C. Lovadina, Analysis of a mixed finite element method for the Reissner-Mindlin plate problems, Comput. Methods Appl. Mech. Engrg., 163(1998), pp. 71-85. MR 2000g:74076

[39] R. Durán and E. Liberman, On the convergence of a triangular mixed finite element method for Reissner-Mindlin plates, M3AS Math. Models Methods Appl. Sci., 6(1996), pp. 339-352. MR 97e:73064

[40] H.-Y. Duan, Studies On Mixed Finite Element Methods, Ph.D. thesis, Institute of Mathematics, Academy of Mathematics and System Sciences, Chinese Academy of Sciences, Beijing, March, 2002.

Institute of Mathematics, Chinese Academy of Sciences, Beijing 100080, Peoples RePUBLIC OF CHINA

E-mail address: dhymath@yahoo.com.cn, hyduan@lsec.cc.ac.cn

Institute of Mathematics, Chinese Academy of Sciences, Beijing 100080, Peoples RePUBLIC OF CHINA

E-mail address: guoping@math03.math.ac.cn, ling@fegensoft.com 